\title{
Ground-based variability surveys towards Centaurus A: worthwhile or not?^
}

\author{
J. T. A. de Jong ${ }^{1,2}$, K. H. Kuijken ${ }^{3,2}$, and P. Héraudeau ${ }^{4,2}$ \\ 1 Max-Planck-Institut für Astronomie, Königstuhl 17, 69117 Heidelberg, Germany \\ e-mail: de jong@mpia.de \\ 2 Kapteyn Astronomical Institute, University of Groningen, PO Box 800, 9700 AV, Groningen, The Netherlands \\ 3 Sterrewacht Leiden, University of Leiden, PO Box 9513, 2300 RA, Leiden, The Netherlands \\ 4 Argelander Institut für Astronomie, Auf dem Hügel 71, 53121 Bonn, Germany
}

Received 30 July 2007 / Accepted 28 November 2007

\section{ABSTRACT}

\begin{abstract}
Context. Difference imaging has proven to be a powerful technique for detecting and monitoring the variability of unresolved stellar sources in M 31. Using this technique in surveys of galaxies outside the Local Group could have many interesting applications. Aims. The goal of this paper is to test difference imaging photometry on Centaurus A, the nearest giant elliptical galaxy, at a distance of $4 \mathrm{Mpc}$.

Methods. We obtained deep photometric data with the Wide Field Imager at the ESO/MPG $2.2 \mathrm{~m}$ at La Silla spread over almost two months. Applying the difference imaging photometry package DIFIMPHOT, we produced high-quality difference images and detected variable sources. The sensitivity of the current observational setup was determined through artificial residual tests.

Results. In the resulting high-quality difference images, we detect 271 variable stars. We find a difference flux detection limit corresponding to $m_{R} \simeq 24.5$. Based on a simple model of the halo of Centaurus A, we estimate that a ground-based microlensing survey would detect in the order of 4 microlensing events per year due to lenses in the halo.

Conclusions. Difference imaging photometry works very well at the distance of Centaurus A and promises to be a useful tool for detecting and studying variable stars in galaxies outside the local group. For microlensing surveys, a higher sensitivity is needed than achieved here, which would be possible with a large ground-based telescope or space observatory with wide-field imaging capabilities.
\end{abstract}

Key words. galaxies: individual: Centaurus A - galaxies: stellar content - stars: variables: general - gravitational lensing

\section{Introduction}

Using difference imaging techniques (Tomaney \& Crotts 1996; Gould 1996; Alard 2000) it is possible to detect and monitor stellar variability in highly crowded fields where the individual stars are unresolved. During the past decade these techniques have been used to this aim by several microlensing surveys towards the Magellanic Clouds (e.g. Alcock et al. 2000; Tisserand et al. 2007) and the Andromeda galaxy (M 31) (e.g. Riffeser et al. 2003; Calchi Novati et al. 2005; de Jong et al. 2006). This has led to the successful detection of several microlensing events and tens of thousands of previously unknown variable stars in M 31. Following these results the question arises whether the application of these techniques can be extended to even more distant objects. Both the detection of variable stars and of microlensing in galaxies outside the local group would have several interesting applications.

The number of known variable stars in galaxies could be multiplied, which would be interesting in itself, and also useful for, for example, distance determination and the study of stellar populations and star formation histories. Microlensing surveys outside the Local Group would also be an important tool to study the luminosity functions and the halo compact object content of galaxies other than the Milky Way and M 31. On the one hand

* Catalogue is only available in electronic form at the CDS via anonymous ftp to cdsarc.u-strasbg.fr (130.79.128.5) or via http://cdsweb.u-strasbg.fr/cgi-bin/qcat?J/A+A/478/755 this would be useful as a further measure of the contribution of massive compact halo objects (MACHOs) to galaxy halos. On the other hand such microlensing surveys could be an important step to the generalisation of the picture obtained from the surveys in the Local Group. Both the Milky Way and M31 are two large spiral galaxies in a relatively low-density environment. It would be interesting to also study the compact object content in the halos of galaxies in other environments or in the halos of other types of galaxies.

If the dark matter does indeed have a baryonic MACHO contribution, this component could be different in elliptical galaxies compared to spirals, since they are believed to have undergone different formation histories and star formation episodes. Furthermore, elliptical galaxies tend to live in more dense environments, where galactic encounters are more common and old stellar populations would have been pushed out to larger radii into the halo. Microlensing surveys in elliptical galaxies would also enable the study of these stellar halos, that might be important repositories of faint stellar mass.

A first attempt at a microlensing survey in an elliptical galaxy was made by Baltz et al. (2004). Using observations taken with the Wide Field and Planetary Camera 2 (WFPC2) on the Hubble Space Telescope, they performed a search for microlensing events in the giant elliptical galaxy M 87. Due to the large distance to M 87 of about 16 Mpc (Tonry et al. 2001) doing this experiment proved difficult in practice. In their study, Baltz et al. (2004) find only seven variable sources, 1 of which is 
consistent with microlensing, although it is bluer than expected for a typical microlensing event.

In this paper we present a pilot study of a ground-based variability study using difference imaging photometry towards the giant elliptical galaxy Centaurus A (Cen A, NGC 5128). Apart from the general interest of a variable star and microlensing study in an elliptical galaxy discussed above, this survey would have additional scientific interests. From the warped gas layer, the outer isophotes and the kinematics of the planetary nebulae, there are indications that the potential and therefore the halo around Centaurus A is triaxial (Hui et al. 1995). This means that the lines-of-sight toward different parts of the galaxy have different path-lengths through the halo. Because of this, the microlensing event rate will be asymmetric over the face of Cen A, much like the microlensing rate due to halo lensing is asymmetric between the near and far side of the disk of M 31. The spatial distribution of halo microlensing events could therefore also be used to constrain the shape of the dark halo of Cen A. Because the distance to Cen A (4 Mpc, Tonry et al. 2001) is much smaller than the distance to $M 87$, the sensitivity will be much higher and the crowding of variable sources much less strong. Nevertheless, Cen A lies five times further away than M 31, so the project is not without challenge.

Section 2 describes the data and methods that were used in this study. In Sect. 3.2 the resulting difference images are described and the detected variable sources presented. We determine the detection efficiency for variable sources in Sect. 3.3. The prospects of ground-based (halo) microlensing surveys towards Centaurus A are estimated in Sect. 4, based on our results and a simple model of the halo of Centaurus A. Finally, in Sect. 5 we present our conclusions.

\section{Data and methods}

Goal of the observational set-up was to obtain deep, 9000 s, photometry in the broad $R_{\mathrm{c}}$ filter during each of a series of nights spread over a several month period, resulting in both high sensitivity and sufficient temporal coverage to detect faint variable sources. Observations were done during 12 nights spread between April 15th and June 8th, 2005 with the Wide Field Imager (WFI; Baade et al. 1999) at the ESO/MPG $2.2 \mathrm{~m}$ telescope at La Silla, Chile. With its field-of-view of $34^{\prime} \times 33^{\prime}$ (pixel scale $0.238^{\prime \prime}$ per pixel) the WFI enables the monitoring of Cen A in one single pointing. The total exposure time per night was divided over $1000 \mathrm{~s}$ exposures to help with cosmic ray rejection. A small subset of data was discarded because of poor seeing $\left(>1.3^{\prime \prime}\right)$, leaving data for 10 nights, and 9 nights with the total exposure time of $8000 \mathrm{~s}$ or longer. A summary of the exposure times and average seeings is listed in Table 1. The seeings listed are the average of all exposures from the four central chips in the array, with the quoted errors the standard deviation.

The data reduction was performed with the Astronomical Wide-Field Imaging System for Europe ${ }^{1}$ (AstroWISE, Valentijn et al. 2007). A short description of the main steps of the data reduction process is given here.

- Bias subtraction: for each night a master bias image was created from between 10 and 42 raw bias frames after doing an overscan correction and outlier rejection. These master bias frames were subtracted from all overscan-corrected science frames to remove $2 \mathrm{D}$ bias patterns.

1 A detailed description of the system can be found at the AstroWISE portal: www .astro-wise.org/portal
Table 1. Summary of data used.

\begin{tabular}{lccc}
\hline \hline Date & $\begin{array}{c}\text { Exposures } \\
\text { Total } t_{\exp }\end{array}$ & $\begin{array}{c}\text { Seeing } \\
(\mathrm{s})\end{array}$ \\
\hline April 17 2005 & 4 & 4000 & $0.99 \pm 0.06$ \\
April 30 2005 & 9 & 9000 & $0.83 \pm 0.10$ \\
May 9 2005 & 8 & 8000 & $0.78 \pm 0.03$ \\
May 11 2005 & 9 & 9000 & $0.84 \pm 0.13$ \\
May 12 2005 & 9 & 9000 & $0.92 \pm 0.07$ \\
May 29 2005 & 9 & 9000 & $1.00 \pm 0.14$ \\
May 30 2005 & 9 & 9000 & $0.82 \pm 0.10$ \\
May 31 2005 & 9 & 9000 & $0.80 \pm 0.13$ \\
June 1 2005 & 9 & 9000 & $1.02 \pm 0.15$ \\
June 8 2005 & 9 & 9000 & $0.79 \pm 0.07$ \\
\hline
\end{tabular}

Table 2. Mean zero points and their uncertainties.

\begin{tabular}{lcc}
\hline \hline Chip & $\begin{array}{c}\text { Zero point } \\
\text { (mag) }\end{array}$ & $\begin{array}{c}\text { Uncertainty } \\
\text { (mag) }\end{array}$ \\
\hline ccd50 & 24.238 & 0.040 \\
ccd51 & 24.334 & 0.048 \\
ccd52 & 24.113 & 0.040 \\
ccd53 & 24.312 & 0.035 \\
ccd54 & 24.311 & 0.029 \\
ccd55 & 24.363 & 0.028 \\
ccd56 & 24.293 & 0.034 \\
ccd57 & 24.171 & 0.029 \\
\hline
\end{tabular}

- Flat-fielding: master dome and twilight flats were constructed for each night from between 5 to 20 exposures. The master dome and twilight flat-fields are combined into a master flat-field used to correct the science frames.

- Astrometry: the astrometry was derived using routines from the Leiden Data Analysis Center (LDAC). A preliminary astrometry is first estimated as a simple shift of the 8 chips together to align the field with the coordinates in the USNO A2.0 catalogue. The final astrometry for each chip is derived by using 2 nd order polynomials to fit the position of stars detected in all exposures to their USNO coordinates.

- Photometry: since the photometry of the images is scaled to a reference image during the difference imaging procedure, only mean zero points were computed in AstroWISE to convert the ADU flux of variable stars to magnitudes. The zero points were derived as the median of the zero points obtained from the Landolt standard fields observed during the whole period of the observations assuming a mean extinction in $R_{\mathrm{c}}$ of 0.09 mag. On average, 4 standard fields were observed per night, with an average of 100 standard stars landing on each chip. The zero points and their uncertainties are listed in Table 2. The uncertainties are the standard deviations of the zero points derived from all 40 standard field observations.

- Resampling: finally, images were regridded to a pixel scale equal to $0.2^{\prime \prime} \mathrm{pix}^{-1}$ using the LANCZOS3 algorithm in SWARP $^{2}$.

\subsection{Difference imaging photometry}

For the difference image analysis the same pipeline is used as was employed by de Jong et al. (2006) in their analysis of M 31 data. This method is based on the procedures for matching the point spread function (PSF) between two images described by Tomaney \& Crotts (1996) and although it has a different

\footnotetext{
${ }^{2}$ For information on SWARP see http://terapix.iap.fr/
} 

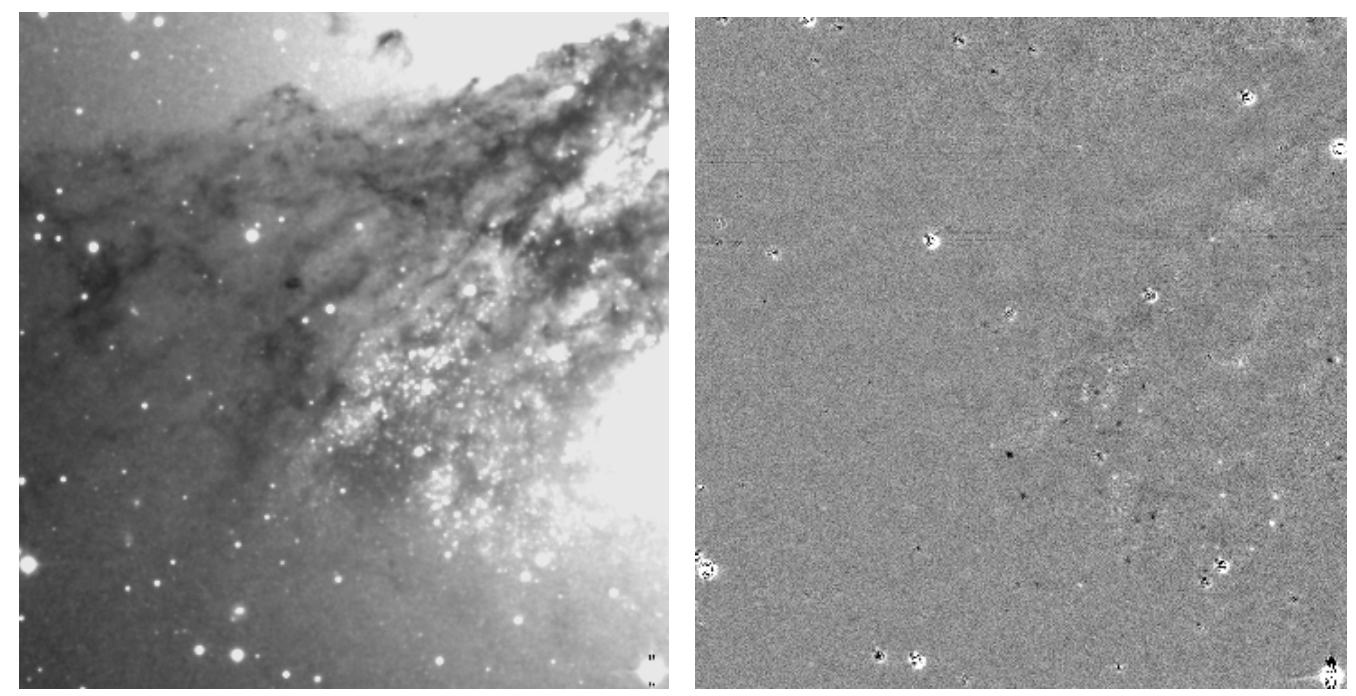

Fig. 1. Example of an area in Centaurus A and its difference image. Left: detail of the reference image showing the dust lanes and star forming regions to the south-east of the centre of Centaurus A. Right: detail of the difference image of June 8th, 2005 showing the same region of Centaurus A.

approach to the problem of PSF-matching of two images from the method developed by Alard (2000) it produces difference images of similar quality. The difference image pipeline runs in IRAF and makes use of the DIFIMPHOT package written primarily by Austin Tomaney. Taking the reduced images as input, the pipeline goes through the following steps.

- The images are aligned to a common astrometric reference frame to an rms precision better than 0.1 pixels using stars spread over the whole image.

- Using cuts on the seeing, astrometric shift and sky brightness, the best $\sim 15$ exposures are selected and combined into a high signal-to-noise reference image.

- All images obtained during the same night are combined into one image. These nightly image stacks will provide the photometric measurements for the detection of variable sources and their light-curves. Thus, since we use data obtained during 10 nights we have 10 temporal epochs in the subsequent analysis.

- After photometric calibration and matching of the PSF the reference image is subtracted from each nightly stack, resulting in a difference image for each night. The PSF of the nightly stacks and the reference image is measured from bright but unsaturated stars on the image. From these PSFs a convolution kernel is derived in Fourier space to convert the better seeing image to the seeing of the worst seeing image before subtraction. To cope with PSF variations across the chips, each chip is divided in 16 subregions, for each of which the PSF matching is done separately, using a minimum of 10 stars. Photometric calibration is done using a subset of non-variable bright stars. The resulting difference images are dominated by shot noise in which variable sources show up as positive or negative residuals.

- Variable sources are detected on the difference images using SExtractor (Bertin \& Arnouts 1996), where we select groups of 3 adjacent pixels that are at least $3 \sigma$ above or below the local background. Bright stars, saturation spikes and bad pixels are masked out during this step.

- Using PSF-fitting photometry the brightness of all variable sources is measured from the difference images and lightcurves are constructed.

\section{Results}

\subsection{Difference image quality}

The difference images that result from the above described procedure are of very high quality, with noise levels close to the theoretical photon noise. In Fig. 1 a cutout from the reference image and the corresponding cutout from the difference image of June 8th, 2005 are shown. While most of the difference image shows only noise, variable stars in the star forming regions leave positive and negative residuals. Bright foreground stars also leave residuals in the difference image due to imperfect subtraction.

As shown theoretically by Gould (1996), it is in principle, possible to create difference images where the noise is dominated by photon noise. Noise due to systematic effects such as PSF variation and geometric and photometric alignment can, when handled carefully, be brought down to levels where they cannot compete with the photon noise. To assess how well our difference images approach the limit of pure photon noise, we have determined the noise in several regions of highly varying background intensity where no obvious variable sources are present. In the upper panel of Fig. 2 these noise levels are plotted versus the background intensity for four different images with different FWHM. There is a strong correllation with higher noise in brighter regions, which is a clear signature of the photon noise. Apart from this, there are offsets between the different epochs, which depend on a combined accuracy of the PSF-matching, photometric and astrometric alignment, seeing variations between individual exposures etc.

The lower panel of Fig. 2 shows the same, but now divided by the theoretical photon noise. For a single exposure, the photon noise is simply the square root of the number of counts in a pixel; in this case both the reference image and the nightly images are stacks of several exposures, so that in each the photon noise should be the square root of the counts divided by the square root of the number of exposures going into the stack. The quality of our difference images turns out to be very good, with the noise very close to the theoretical photon noise. There might be a slight degradation at very high background levels, because very close to the centre of Centaurus $A$ the number of stars that 


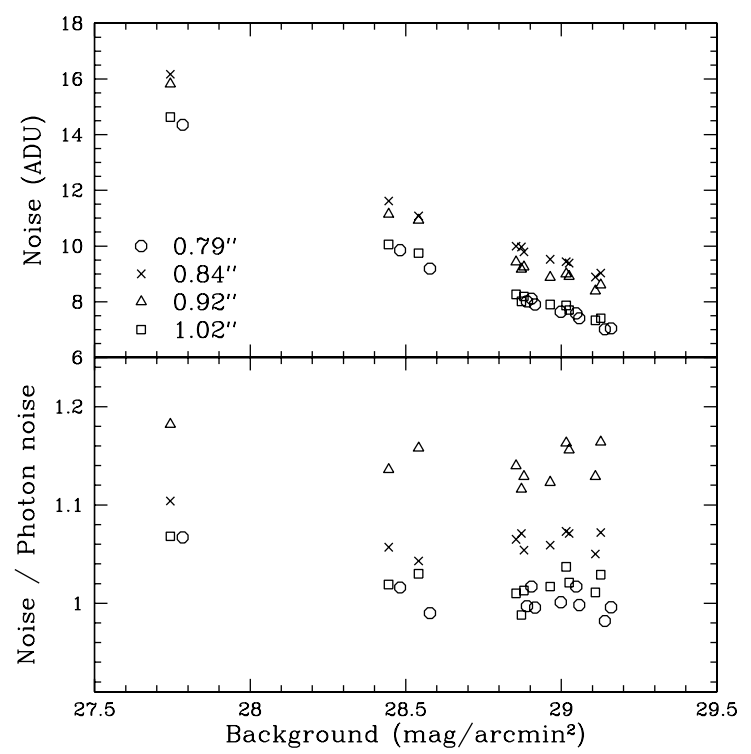

Fig. 2. Difference image noise levels for a set of regions with different background intensity. Top: standard deviation of pixel values for four epochs with different seeing values: May 11, 2005 (0.84", crosses), May 12, 2005 (0.92", triangles), June 1, 2005 (1.02", squares), and June 8, $2005\left(0.79^{\prime \prime}\right.$, circles). Bottom: same as in top panel, but scaled to the theoretical photon noise.

can be used to construct an accurate PSF model is low, due to saturation of the chips.

Another interesting test is to look at the residuals around resolved, bright, but unsaturated stars. Because of their higher brightness, secondary effects such as imperfections in the PSF-matching kernel or photometric scaling will show up more strongly. Figure 3 shows the noise divided by the theoretical photon noise for stars of varying brightness in a high background region $\left(\mu_{R} \sim 28.0 \mathrm{mag} / \mathrm{arcmin}^{2}\right.$, upper panel) and in a low background region $\left(\mu_{R} \sim 29.0 \mathrm{mag} / \mathrm{arcmin}^{2}\right.$, lower panel). In both panels the residuals are much higher than the photon noise for bright stars, and decreasing to the photon noise level at faint magnitudes. In the high background region this decrease is faster because the photon noise is higher. That the residuals at the bright end are higher in the high background region is caused by the poorer PSF modelling near the centre of Centaurus A, due to the lack of sufficiently bright, unsaturated stars. It should be noted that this problem does not affect image subtraction methods based on direct pixel-to-pixel fits, such as the one developed by Alard \& Lupton (1998) and Alard (2000).

\subsection{Variable sources}

Residuals were detected in the difference images using Sextractor and at all positions with at least two detections lightcurves were built. A by-eye inspection of light-curves and difference images to reject spurious detections due to bad pixels or foreground stars, resulted in a catalogue of 271 variable sources. A catalogue (Table 3) with the positions of the variables is published only in electronic form with this article.

The majority of these variables are expected to be Cepheids and long period variables (LPVs) such as Mira variables, since these classes are both common and bright. There is also one Nova in the sample. That we would catch one Nova during its peak is not unexpected considering the Nova rate of $\sim 28$ per year for Centaurus A (Ciardullo et al. 1990). With ten points,

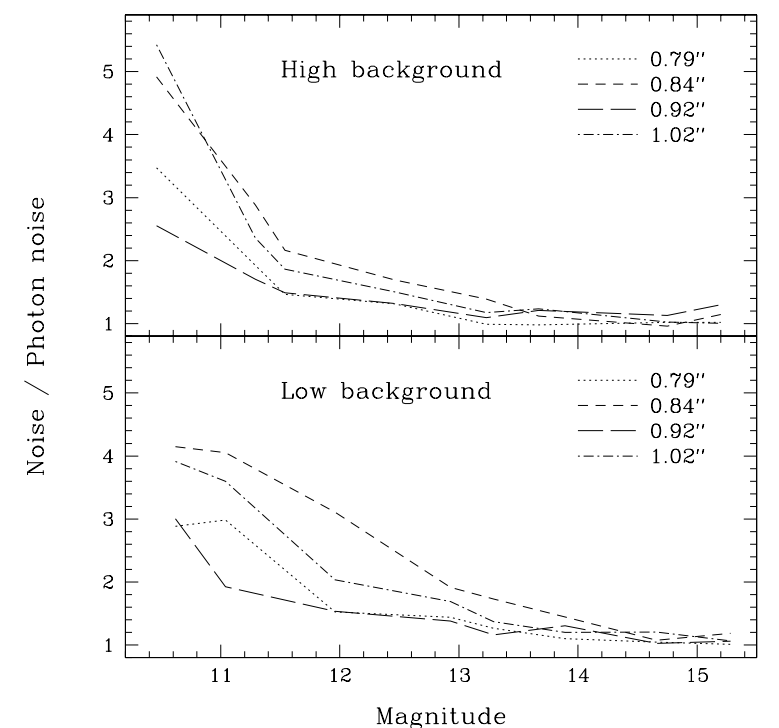

Fig. 3. Residual strength, in terms of theoretical photon noise, of a set of bright, resolved stars of different magnitudes for four observation epochs. Top: residuals in a high surface brightness region $\left(\mu_{R} \simeq\right.$ $28 \mathrm{mag} / \mathrm{arcmin}^{2}$ ) in the difference images of May 11, 2005 (short dashed), May 12, 2005 (long dashed), June 1, 2005 (dot-dashed), and June 8, 2005 (dotted). Bottom: same as top panel, but for a low surface brightness region $\left(\mu_{R} \simeq 29 \mathrm{mag} / \mathrm{arcmin}^{2}\right)$.

the sampling of the light-curves is too sparse to allow an accurate period and amplitude determination for the Cepheids. For the LPVs the situation is worse since their periods are longer than the total time span of our observations. We did, however, apply two different period finding algorithms to the light-curves, namely the multi-harmonic periodogram method described by Schwarzenberg-Czerny (1996) and the normalised periodogram method developed by Lomb (1976), both of which are designed to work on unevenly sampled data. For 29 variables the periods determined with the two methods lie within $10 \%$ of each other and between 2 and 50 days. These limits exclude periods that are longer than the observing period and the aliases that occur close to one day since this is the minimum spacing between the data points. These reasonably robust periods, which are in the range of typical Cepheid periods, are listed in the electronic catalogue of variables (Table 3 ). Of the other 242 variables, the majority of the ones in the star forming regions seem chaotic. These are most likely periodic variables for which the periods cannot be determined because of poor phase coverage. The majority of the variables away from the centre are monotonically increasing or decreasing and can be classified as LPVs.

In Fig. 5 the positions of all 271 variable sources are plotted. There is a clear concentration of variables in the star forming regions in the dust ring in the central parts of Centaurus A. The majority of the stars with confirmed periods between 2 and 50 days are located in these regions. This distribution is not unexpected, since Cepheids are massive stars with masses of at least 3 or 4 times that of the Sun and up to several tens of solar masses, and therefore quite young. On the other hand, LPVs belong to old stellar populations and are expected to be more evenly distributed. Also shown in Fig. 5 are the locations of the fields monitored for variability by Rejkuba et al. (2003) and Ferrarese et al. (2007). Using ISAAC at the Very Large Telescope (VLT), Rejkuba et al. (2003) were able to detect 1504 LPVs in an area just over 10 square arcmin. None of these are detected in our survey, due to the lower sensitivity of our data and the fact 

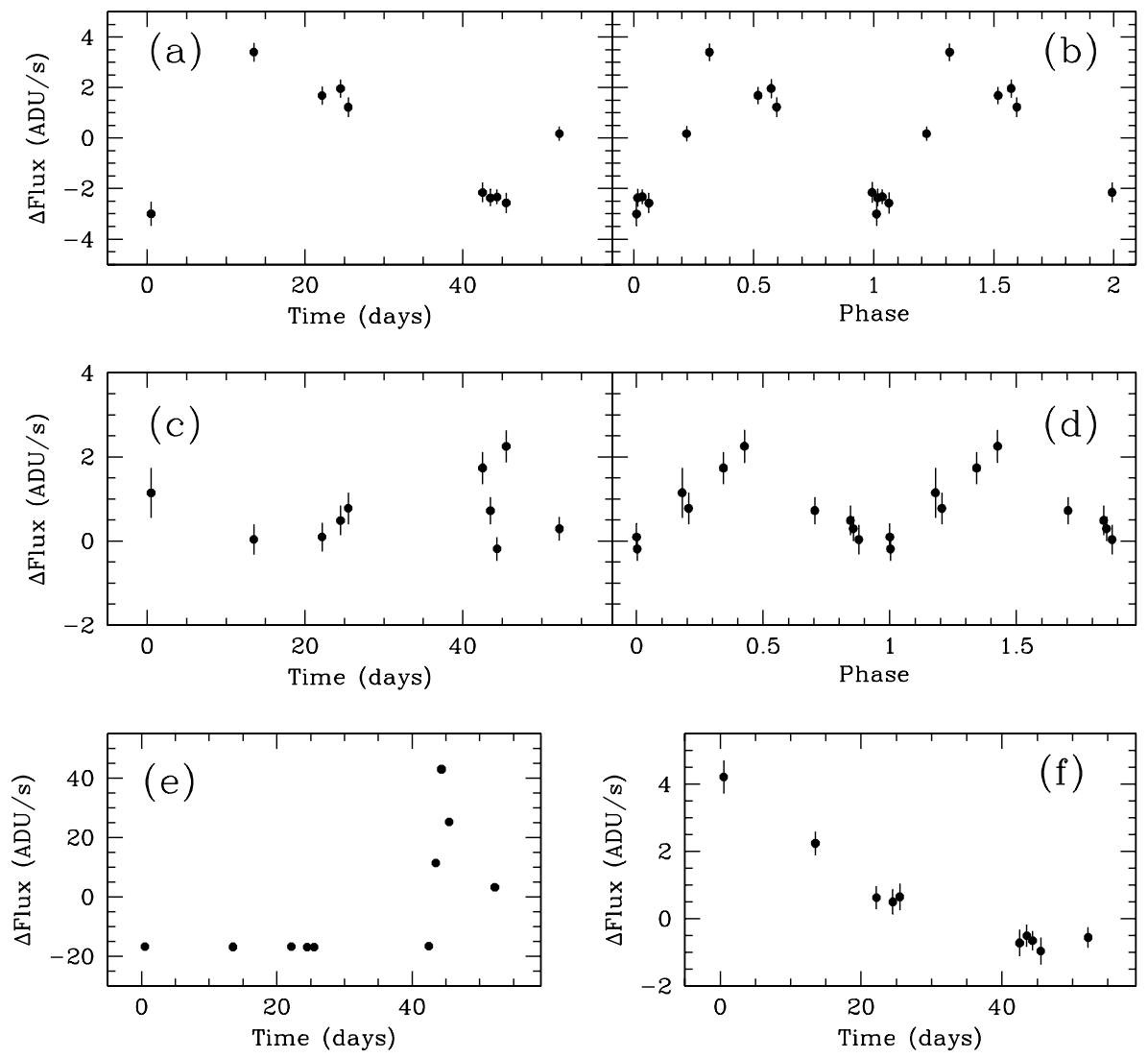

Fig. 4. Selection of light-curves from variable stars in Centaurus A, showing difference flux in $\mathrm{ADU} / \mathrm{s}$ as a function of the time in days from the start of the observations on April 17th, 2005. a) Bright Cepheid-like variable with a period of 43 days. b) Same variable as in a) but showing two periods of the folded lightcurve. c) Cepheid-like variable with a period of 2.8 days. d) Same variable as in c) but showing two periods of the folded light-curve. e) Nova variable, erupting on May 29 and reaching its maximum around May 31, 2005. f) Longperiod variable reaching its minimum at the beginning of June 2005, with the maximum occurring outside our time range.

that LPVs are significantly fainter at optical wavelengths compared to the infrared. Ferrarese et al. (2007) used the Wide Field and Planetary Camera 2 (WFPC2) on board the Hubble Space Telescope to detect Cepheid variables, resulting in a sample of 56 Cepheids and 70 other periodic variables. Due to the difference in sensitivity and the fact that Ferrarese et al. (2007) use clear periodicity as one of their detection criteria, we only identify three stars that are both in our and in their sample. For completeness, also the Nova variables from the extragalactic part of the General Catalogue of Variable Stars (Artyukhina et al. 1996) are plotted. To give the reader an impression of the quality of the light-curves, we present the light-curves of two periodic variables, a Nova and an LPV in Fig. 4. The Nova variable shown in panel (e) of the figure has a peak difference flux corresponding to $m_{R}=20.2$, making this by far the brightest variable in our sample. A period-amplitude plot is shown in Fig. 6 for the 29 variables with robust periods. The amplitudes were determined by taking the difference between the highest and the lowest point of the lightcurve; these amplitudes estimates are lower limits since the actual maxima and minima might not be sampled by the data. The dashed line indicates the period-amplitude relation found for type I Cepheids in M 31 using the same techniques as employed here (de Jong 2005).

\subsection{Detection efficiency}

To determine the detection efficiency for variable sources we put artificial residuals in the difference images and try to "detect" them. Of course, there are different ways to detect sources in variability studies such as pixel lensing surveys. The method used in this paper to detect variable stars searches the difference images for groups of pixels that exceed a certain threshold above or below the background. This method was also used

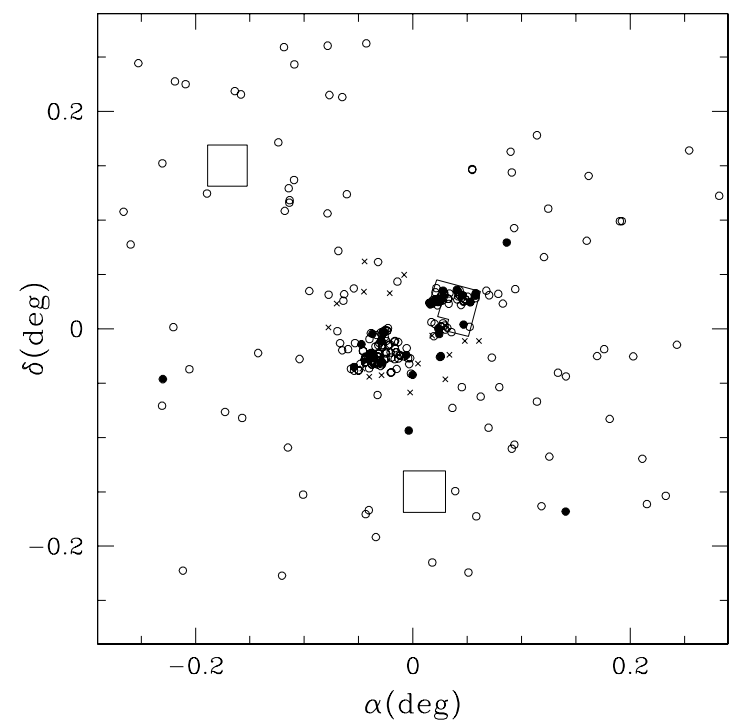

Fig. 5. Positions of the 271 variable sources detected in our survey, with respect to the centre of Centaurus A. Closed circles indicate variable sources for which a reasonably accurate period could be determined. The two squares outline the fields in which Rejkuba et al. (2003) detected a total of 1504 long-period variables using ISAAC at the VLT; the WFPC 2 field-of-view used by Ferrarese et al. (2007) in their survey for Cepheids is located just to the right from the centre of the figure. Crosses indicate the positions of Nova variables taken from Artyukhina et al. (1996).

by the MEGA collaboration in their M 31 microlensing survey (cf. de Jong et al. 2006). In another frequently used method, light-curves are built at each pixel position in the image, after which the light-curves are identified that show a variable signal 


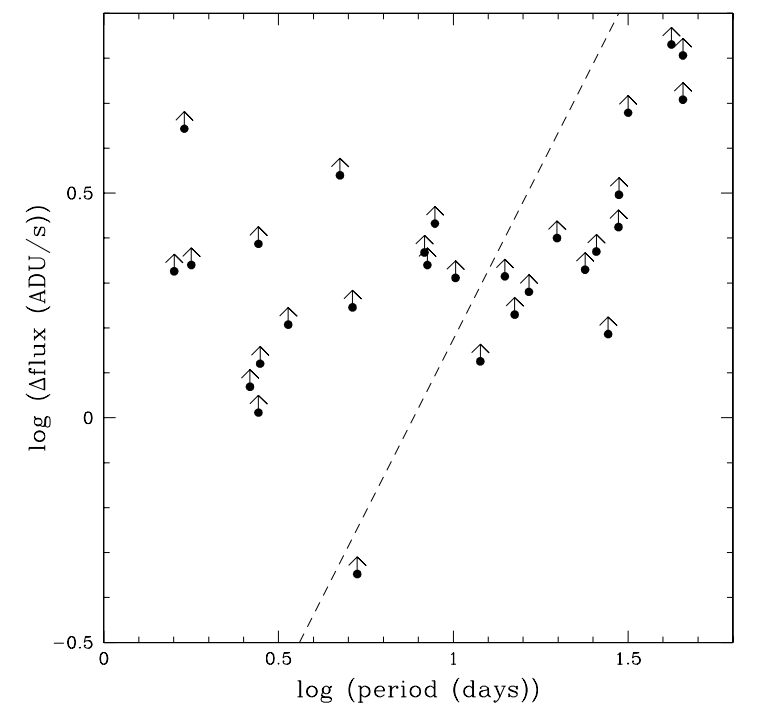

Fig. 6. Periods and amplitude estimates of the 29 variables with robust period determinations. The dashed line represents the approximate period amplitude relation found for type I Cepheids in M 31 (de Jong 2005), scaled to the distance of Centaurus A and corrected for the different gains.

(cf. Calchi Novati et al. 2005). To be as general as possible we will use both approaches in our detection efficiency analysis and show that the difference in sensitivity between the two is small.

We select a subset of six difference images for the detection efficiency tests that are of high quality and from CCDs in the centre of the array. The images are from the nights of April 30, May 30 and June 8, 2005 and correspond to chips 2 and 7 from the CCD array. This way reductions in sensitivity due to poor seeing and PSF degradation in the perifery of the focal plane are avoided. Although these kind of effects are unavoidable in real surveys, the detection efficiencies we retrieve will represent upper limits for the current observational setup. Because the background from unresolved stars varies strongly in intensity across the field, the detection efficiency will vary spatially. To probe this effect we divide both chips in the part where the background surface brightness is lower than $\mu_{R}=28.5 \mathrm{mag} / \mathrm{arcmin}^{2}$ and the part where it is higher, and consider these regions separately. The high background region includes the small but very bright centre of Centaurus A. We randomly place residuals of a fixed (difference) flux in the selected difference images, 1000 at a time in the low background regions and 250 at a time in the smaller high background regions. Fluxes probed are 2, 1.5, 1, 0.75, 0.5, and $0.25 \mathrm{ADU} / \mathrm{s}$, corresponding to 23.6, 24.0, 24.4, 24.7, 25.2 and $25.9 \mathrm{mag}$; in total we put 20000 residuals with these fluxes in each region, respectively. The actual PSFs measured from the stacked nightly images, which are divided in subregions to deal with possible PSF variations across the field, are used for building the residuals. Photon noise is included in the residuals.

After inserting the residuals, SExtractor is run on the difference images and groups of 3 adjacent pixels that are at least $3 \sigma$ above the local background are selected. Comparison of the list of detected sources with the input list of residuals gives the detection efficiency of this residual detection method. At each position where an artificial residual is inserted, we also perform PSF-fitting photometry to measure the flux at that position. We consider the residual to be detected if the recovered flux is at least $3 \sigma$ above zero, where the $\sigma$ is the photometry error. This error is calculated by the PSF-fitting photometry routine and based on the statistics of the fit and includes a correction for

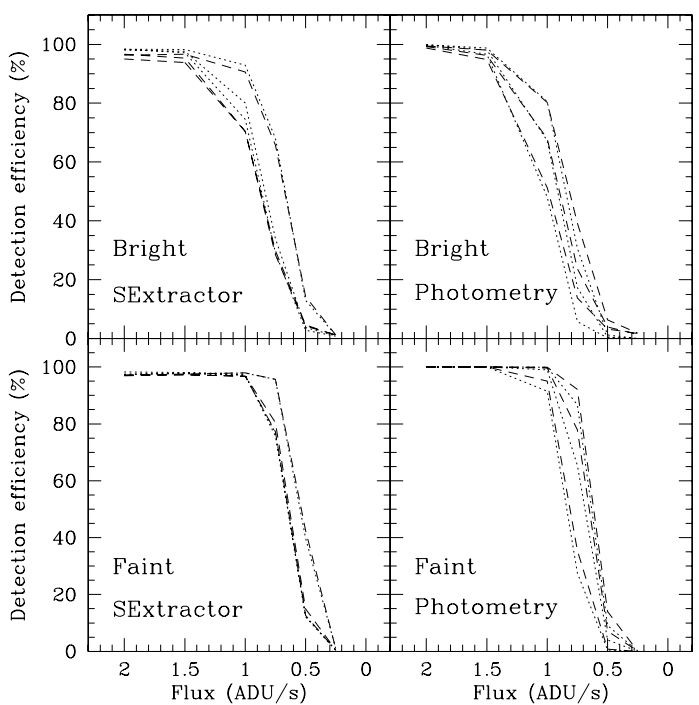

Fig. 7. Detection efficiencies in percent as function of difference flux in ADU/s. Dashed lines show the results for chip 7 in the WFI array, dotted lines those for chip 2, where each line corresponds to a different epoch. The left panels show the efficiencies for the case where residuals are detected as groups of significant pixels in the difference images, the right panels for the case of PSF-fitting photometry. In the upper panels the results for the regions with a background surface brightness higher than $28.5 \mathrm{mag} / \mathrm{arcmin}^{2}$ are plotted, in the lower panels for the regions with lower surface brightness.

photometric scaling inaccuracies during the image subtraction. The fraction of sources detected with this PSF-fitting photometry gives a second measurement of the detection efficiency.

Figure 7 shows the detection efficiencies for both detection methods and all regions. There is indeed a difference in the detection efficiency in the bright regions (upper panels) and the fainter regions (lower panels), although not very large. In both cases the detection efficiency is $100 \%$ down to a flux of $1.5 \mathrm{ADU} / \mathrm{s}$ and virtually $0 \%$ at $0.5 \mathrm{ADU} / \mathrm{s}$, with the transition more gradual in the bright regions. This more gradual slope is due to the larger variation in the background intensity. These results show that even very close to the bright centre of Centaurus A, residuals with a flux of 1.5-1 ADU/s are still detected with high efficiency. There is not much difference between the SExtractor-based detection method (left panels) and the photometry-based method (right panels). A variability survey with the WFI at the ESO/MPG 2.2 m telescope with $9000 \mathrm{~s}$ exposure time per epoch thus will have a detection limit of at best slightly below $1 \mathrm{ADU} / \mathrm{s}$. This corresponds to $\sim 24.5 \mathrm{~m}_{R}$, or for a variable of absolute magnitude $M_{R}=-4$ to a magnitude difference in the order of $1 \mathrm{mag}$. Since the majority of Cepheids has amplitudes of less than 1 mag this means that only the very bright ones or ones with high amplitudes are detected.

\section{Implications for microlensing surveys}

Now that we have shown that Difference Image Photometry works well at the distance of Centaurus A, we want to investigate the prospects of ground-based microlensing surveys towards this galaxy. In the previous section the detection efficiency for a single residual of a certain flux in a high quality difference image was determined. Taking this as the detection efficiency for a microlensing event of the same maximum amplitude is of course a gross oversimplification. To be able to "detect" a microlensing event, enough information must be obtained from the 
light-curve to be reasonably certain of the lensing nature of the detected variability. In practice one would want to detect the event in more than one difference image and with sufficient signal-to-noise to recover certain characteristic signatures, such as light-curve shape and achromaticity. Even assuming a survey with observations every night, a detection limit of $1 \mathrm{ADU} / \mathrm{s}$ is optimistic, but can serve as an upper limit.

In the light of the arguments discussed in Sect. 1 we concentrate on microlensing by objects in the halo of Centaurus A. If we assume the halo is a cored isothermal sphere with velocity dispersion $\sigma$ and core radius $r_{\mathrm{c}}$, the density is given by,

$\rho(r)=\frac{\sigma^{2}}{2 \pi G} \frac{1}{r^{2}+r_{\mathrm{c}}^{2}}$.

By definition, a source is considered to be microlensed if it is lying within the Einstein radius of a lens, i.e. if it is magnified at least a factor of 1.34. The microlensing optical depth $\tau$ is defined as the number of lenses within one Einstein radius of the line-ofsight towards the source and therefore gives the fraction of stars that is microlensed at a given time. This means that $\tau$ is given by,

$\tau=\int_{0}^{r_{\mathrm{h}}} n_{\mathrm{l}}(r) \pi R_{\mathrm{E}}^{2} \mathrm{~d} r=\int_{0}^{r_{\mathrm{h}}} \frac{f_{\mathrm{b}} \rho(r)}{M_{\mathrm{lens}}} \frac{4 \pi G M_{\mathrm{lens}}}{c^{2}} \frac{D_{\mathrm{LS}} D_{\mathrm{OL}}}{D_{\mathrm{OS}}} \mathrm{d} r$,

where $n_{1}$ is the number density of lenses, $f_{\mathrm{b}}$ is the fraction of halo mass in the lenses, $R_{\mathrm{E}}$ is the Einstein radius, and $D_{\mathrm{OL}}, D_{\mathrm{OS}}$ and $D_{\mathrm{LS}}$ are the distances between observer, lens and source. We integrate from the position of the source to the outer radius of the halo $r_{\mathrm{h}}$. For external galaxies like Centaurus $\mathrm{A}, D_{\mathrm{OL}} \simeq D_{\mathrm{OS}}$ and $D_{\mathrm{LS}} \simeq r$, so that,

$\tau\left(r_{0}\right) \sim \frac{2 f_{\mathrm{b}} \sigma^{2}}{c^{2}} \int_{0}^{\sqrt{r_{\mathrm{h}}^{2}-r_{0}^{2}}} \frac{r}{r^{2}+r_{0}^{2}+r_{\mathrm{c}}^{2}} \mathrm{~d} r$,

for a source star located at a projected distance $r_{0}$ from the centre of the galaxy. Integrating gives,

$\tau\left(r_{0}\right)=\frac{f_{\mathrm{b}} \sigma^{2}}{c^{2}} \ln \left(\frac{r_{\mathrm{h}}^{2}+r_{\mathrm{c}}^{2}}{r_{0}^{2}+r_{\mathrm{c}}^{2}}\right)$.

For a MACHO mass fraction $f_{\mathrm{b}}$ of $10 \%$, a halo velocity dispersion of $\sim 100 \mathrm{~km} \mathrm{~s}^{-1}$ (Peng et al. 2004) and reasonable values for $r_{\mathrm{h}}, r_{0}$ and $r_{\mathrm{c}}$ this gives values in the order of $10^{-7}$, meaning that at any time one in ten million stars is being microlensed.

However, at the distance of Centaurus A we are in the regime where only very high amplification events are detected, the socalled spike pixel-lensing regime defined by Gould (1996). In this regime the impact parameter $\beta$, expressed in units of the Einstein Radius $R_{\mathrm{E}}$, is very small, $\beta \ll 1$, and inversely proportional to the maximum amplification $A: \beta \sim 1 / A$. Suppose a source has to change in brightness by a certain amount $L_{\text {det }}$ in order to be detected. This means that for a microlensing event to be detected, the maximum impact parameter is $\beta \sim 1 / A=L / L_{\operatorname{det}}$ for a source star with luminosity $L$. The number of detectable microlensing events at any time is now given by the optical depth multiplied by the square of the impact parameter,

$N_{\mathrm{det}} \simeq \tau \cdot \beta^{2}(L)=\tau \int \mathrm{d} L f(L) \frac{L^{2}}{L_{\mathrm{det}}^{2}}$,

where $f(L)$ is the luminosity function of the source stars. Using the fact that the luminosity of the galaxy $L_{\mathrm{gal}}=\int \mathrm{d} L f(L) L$ and the fluctuation brightness of the stellar population $\bar{L}=$ $\int \mathrm{d} L f(L) L^{2} / L_{\mathrm{gal}}$ we obtain,

$N_{\mathrm{det}} \simeq \tau \frac{\bar{L} L_{\mathrm{gal}}}{L_{\mathrm{det}}^{2}}$

The absolute magnitude of Centaurus $\mathrm{A}$ is $M_{R} \simeq-22$ (Lauberts $\&$ Valentijn 1989) and a typical value for the fluctuation brightness of an old stellar population is in the order of $R \simeq-1.3$ (e.g. Buzzoni 1993; Tonry et al. 2001). Given our detection limit of $m_{R} \simeq 24.5$, corresponding to $M_{R} \simeq-3.5$ this leads to a number of detectable microlensing events at any time of $N_{\text {det }}=10^{6.5} \tau$. Assuming a MACHO halo mass fraction of about $10 \%$ this means that at any moment $\sim 0.3$ microlensing events can be seen.

The rate $\Gamma$ at which microlensing events due to halo lensing occur is given by the number of events $N_{\text {det }}$ divided by the average duration of the events, measured by the time during which the magnification is above half the maximum magnification $t_{\mathrm{FWHM}}$. In the regime where $\beta \ll 1$, we can approximate $t_{\mathrm{FWHM}}=\sqrt{3} \beta t_{\mathrm{E}}$ (Gondolo 1999). Here $t_{\mathrm{E}}$ is the Einstein crossing time, the time it takes the lens to cross the full Einstein disk, given by,

$t_{\mathrm{E}}=\frac{2 R_{\mathrm{E}}}{v_{\perp}}=\frac{2}{v_{\perp}} \sqrt{\frac{4 G M_{\text {lens }}}{c^{2}} \frac{D_{\mathrm{LS}} D_{\mathrm{OL}}}{D_{\mathrm{OS}}}}$
$\simeq 2.8 \times 10^{7} \mathrm{~s}\left(\frac{M}{M_{\odot}}\right)^{1 / 2}\left(\frac{D_{\mathrm{LS}}}{10 \mathrm{kpc}}\right)^{1 / 2}\left(\frac{v_{\perp}}{100 \mathrm{~km} \mathrm{~s}^{-1}}\right)^{-1}$,

where $v_{\perp}$ is the transverse velocity of the lens with respect to the observer-source line of sight. A typical value of $t_{\mathrm{E}}$ for $1 M_{\odot}$ halo objects in Centaurus A is therefore in the order of a year. The event rate is now given by,

$$
\begin{aligned}
\Gamma & \simeq \tau \int \mathrm{d} L f(L) \beta^{2} / t_{\mathrm{FWHM}} \simeq \sqrt{3} \frac{\tau}{t_{\mathrm{E}}} \int \mathrm{d} L f(L) \frac{L}{L_{\mathrm{det}}} \\
& \simeq \sqrt{3} \frac{\tau}{t_{\mathrm{E}}} \frac{L_{\mathrm{gal}}}{L_{\mathrm{det}}},
\end{aligned}
$$

and filling in the numbers gives $\Gamma \simeq \sqrt{3} \times 10^{7.4} \tau / t_{\mathrm{E}}$. Again assuming an $f_{\mathrm{b}}$ of $10 \%$ we get an expected detectable event rate of $\sim 4$ per year. The average event duration is simply $N_{\text {det }} / \Gamma \sim$ 1 month.

An added complication for studying microlensing due to MACHOs in the halo of Centaurus A is that these microlensing events have to be separated from different possible contaminants, such as variable stars and lensing by other lens populations. Most periodic variables can be recognised as such by extending the time baseline of the survey sufficiently. Cataclysmic variables are not period, but can be identified based on the lightcurve shape and colour evolution. Considering the expected Nova rate for Centaurus A of 28 per year (Ciardullo et al. 1990), which is several factors higher than the halo microlensing rate, the need for sufficiently dense time sampling and multi-band photometry is clear. Microlensing by stars in Centaurus A, rather than by objects in the halo, will also have to be disentangled from the halo lensing signal. As for M 31 and M 87, this so-called "selflensing" will dominate over halo lensing in the central parts of Centaurus A (cf. Baltz et al. 2003, 2004; Riffeser et al. 2006). The cleanest halo lensing sample is therefore obtained in the outer parts of the galaxy.

\section{Conclusions}

In this paper we have demonstrated that difference imaging techniques can be successfully applied to ground-based data of 
Centaurus A, a giant elliptical galaxy at a distance of $4 \mathrm{Mpc}$. Using the Wide Field Imager at the ESO/MPG $2.2 \mathrm{~m}$ telescope at La Silla, and a total exposure time of $9000 \mathrm{~s}$ per epoch, we reach a difference flux detection limit corresponding to a brightness of $m_{R} \simeq 24.5$. We have detected and photometered 271 variable sources in Centaurus A, mostly Cepheids and long-period variables and a Nova. The majority of these variables were previously unknown and are available as an online catalogue with this paper. Clearly, these techniques can be used as powerful tools to enlarge the samples of known variable stars in and to study stellar populations of Centaurus A and other galaxies in the nearby universe. At these depths the difference images do not suffer from crowding, and can be photometered straightforwardly.

The microlensing optical depth towards Centaurus A due to lenses in the halo is of the order of $\tau \sim 10^{-7}$ if we assume that $10 \%$ of the halo mass is in compact objects. Given this optical depth and our detection limit the expected microlensing event rate is $\sim 4$ per year over the whole galaxy. At large distances it will be impossible to identify the source stars of individual microlensing events and blending by stars and variables that are too faint to be detected will play a more important role. Also, only very high amplification events can be observed, meaning that the microlensing is completely in the spike regime (Gould 1996). Even more than for microlensing in M 31, the information that can be obtained for individual events is limited. Statistical analysis of event rates, spatial distribution and timescale distribution is the only way of extracting scientific information out of these data. This again emphasises the need for large samples of microlensing events.

The pilot experiment described here shows that it would be possible to put useful limits on the number of microlensing events in Centaurus A, but a survey that is several magnitudes deeper would be required. This could be achieved with a large ground-based telescope equipped with a wide-field imager large enough to take in all of the galaxy in one exposure, or better still, with a wide-field space observatory. We therefore endorse the plea of Baltz (2005) for inclusion of microlensing of nearby galaxies into the observing programmes of upcoming wide-field cosmological survey facilities.
Acknowledgements. We thank the anonymous referee for useful comments and suggestions to improve this paper. J.T.A.d.J. acknowledges support from DFG Priority Program 1177. Ph. Héraudeau acknowledges the financial support provided through the European Community's Human Potential Programme under contract HPRM-CT-2002-00316, SISCO.

\section{References}

Alard, C. 2000, A\&AS, 144, 363

Alard, C., \& Lupton, R. H. 1998, ApJ, 503, 325

Alcock, C., Allsman, R. A., Alves, D. R., et al. 2000, ApJ, 542, 281

Artyukhina, N. M., Durlevich, O. V., Frolov, M. S., et al. 1996, VizieR Online Data Catalog, 2205

Baade, D., Meisenheimer, K., Iwert, O., et al. 1999, Messenger, 95, 15

Baltz, E. A. 2005, ApJ, 624, 168

Baltz, E. A., Gyuk, G., \& Crotts, A. P. S. 2003, ApJ, 582, 30

Baltz, E. A., Lauer, T. R., Zurek, D. R., et al. 2004, ApJ, 610, 691

Bertin, E., \& Arnouts, S. 1996, A\&AS, 117, 393

Buzzoni, A. 1993, A\&A, 275, 433

Calchi Novati, S., Paulin-Henriksson, S., An, J., et al. 2005, A\&A, 443, 911

Ciardullo, R., Tamblyn, P., Jacoby, G. H., Ford, H. C., \& Williams, R. E. 1990, AJ, 99, 1079

de Jong, J. T. A. 2005, Ph.D. Thesis, University of Groningen, The Netherlands de Jong, J. T. A., Widrow, L. M., Cseresnjes, P., et al. 2006, A\&A, 446, 855

Ferrarese, L., Mould, J. R., Stetson, P. B., et al. 2007, ApJ, 654, 186

Gondolo, P. 1999, ApJ, 510, L29

Gould, A. 1996, ApJ, 470, 201

Hui, X., Ford, H. C., Freeman, K. C., \& Dopita, M. A. 1995, ApJ, 449, 592

Lauberts, A., \& Valentijn, E. A. 1989, The surface photometry catalogue of the ESO-Uppsala galaxies (Garching: European Southern Observatory)

Lomb, N. R. 1976, AP\&SS, 39, 447

Peng, E. W., Ford, H. C., \& Freeman, K. C. 2004, ApJ, 602, 685

Rejkuba, M., Minniti, D., \& Silva, D. R. 2003, A\&A, 406, 75

Riffeser, A., Fliri, J., Bender, R., Seitz, S., \& Gössl, C. A. 2003, ApJ, 599, L17

Riffeser, A., Fliri, J., Seitz, S., \& Bender, R. 2006, ApJS, 163, 225

Schwarzenberg-Czerny, A. 1996, ApJ, 460, L107

Tisserand, P., Le Guillou, L., Afonso, C., et al. 2007, A\&A, 469, 387

Tomaney, A. B., \& Crotts, A. P. S. 1996, AJ, 112, 2872

Tonry, J. L., Dressler, A., Blakeslee, J. P., et al. 2001, ApJ, 546, 681

Valentijn, E. A., McFarland, J. P., Snigula, J., et al. 2007

[arXiv:astro-ph/0702189] 